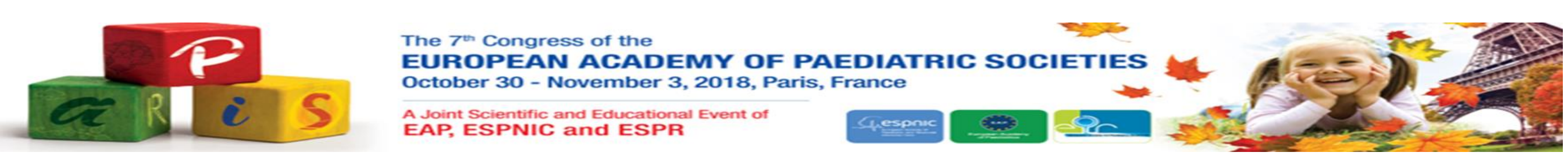

\title{
Cysto-bronchial fistula recurrence: postoperative complications of a pulmonary hydatid cyst (therapeutic approach)
}

Auteurs: Hatri.F, Bouanani.I; Rahmouni.F; Hanou.M; Ouadah.Y; Yazi.AE Service de chirurgie pédiatrique CHU Sidi Bel Abbes /Algérie

\section{Introduction}

Hydatid disease of the lungs is endemic in Algeria with an incidence of 3.4 to 4.6 cases / 100,000 inhabitants.

Surgical treatment is often conservative, however the problem lies in the recurrence of pneumothorax by reversal of bronchial cystic fistulas in complicated forms.

Our objective is to evaluate the frequency of this postoperative complication and to determine these risk factors.

\section{Materials and method}

Monocentric retrospective study from 2007 to 2016 of a series of 60 children treated for pulmonary Hydatid disease.

All our patients benefited from a conservative surgery, which consisted in killing the parasite and treating the residual cavity with closure of the cysto-bronchial fistulas, associated with a double thoracic drainage. Some patients have postoperatively post-surgery recanalization of bronchial cystic fistulas (pneumothorax).

The therapeutic approach was the simple delivery of the thoracic drainage for some patients, for others it is the pleural decortication or regulated resections of the pulmonary parenchyma.

\section{Results}

The average age of our patients was 7 years with extremes of 2 to 15 years. Male predominance was noted with a sex ratio of 1.5 .

The revelation of the disease was fortuitous in $25 \%$ of cases and polymorphic (cough, vomica, infectious syndrome and hemoptysis) in $75 \%$ of cases.

Post operative results

The postoperative follow-up was favorable in $90 \%$ of cases, morbidity related to persistence of air effusion 06 case related to a recurrence of cysto-bronchial fistulas, observed for infected KHP, giant cysts and poly cystitis pulmonary.

The therapeutic approach:

Prolonged thoracic drainage in 02 cases and 04 cases

Pleural decortication with fistula closure and drainage for encysted PNO (pachy pleuritic).

Fixed resection of friable and infected lung parenchyma

The average length of hospital stay 7 days, range 5 to 21 days.

Long-term monitoring was clinical, radiological and biological (hydatid serology).

\section{Discussion}

Pulmonary localization estimated at $60 \%$ comparable with other series. [1,2]. The treatment surgical must be conservative $[3,4,5]$, is to suture the bronchial fistulas and lay flat the cavity by a resection of the dome salient, it is the method of choice of our service.

The review of the literature is poor concerning this complication. However, some authors advocate total per - cystectomy, that is, resections that are regulated for giant or complicated cysts, with the aim of avoiding the recurrence of pleural air effusion by repealing the cyst bronchial fistulas $[8,9]$.

\section{Conclusion}

The pulmonary hydatid disease of the child is relatively benign, the Dg is essentially radiological, the contribution of the biology is modest.

Conservative surgery is the treatment of choice for the child, although the indications for per-cystectomy, or resection, are found in the complicated forms of pulmonary hydatid disease.

Prophylaxis remains the best way to fight this disease, based on the interruption of the parasite cycle, unfortunately difficult to apply in rural areas.

Table 1: Distribution of Respiratory Signs Table 2: Rx aspect of the KHP in the series
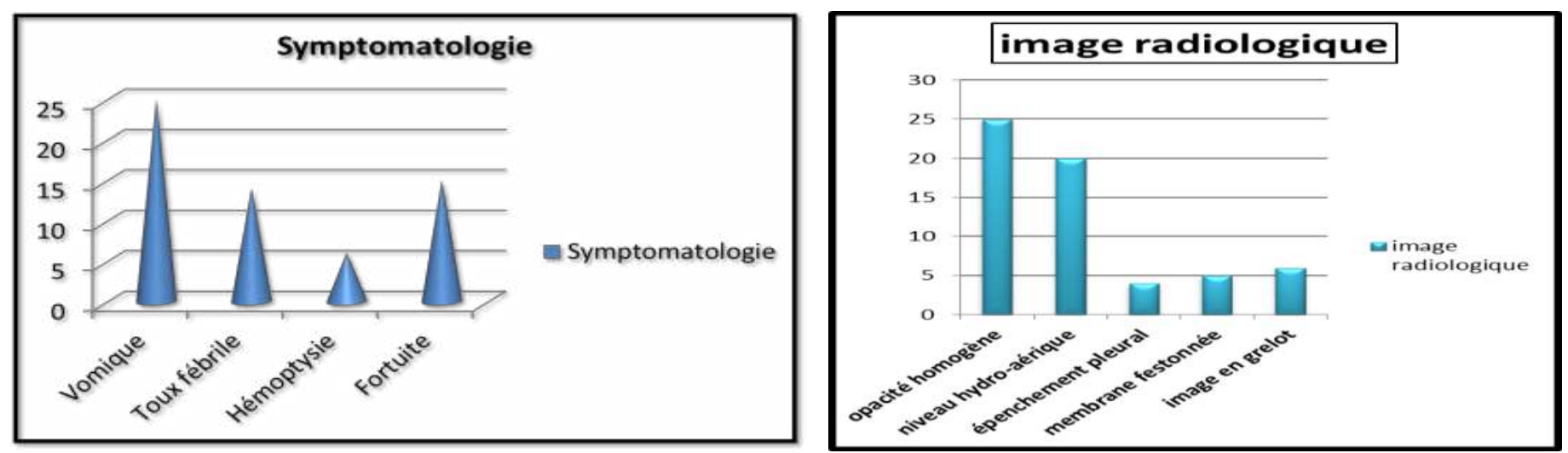

Table 3: Associated locations

Table 4: Topographic Locations

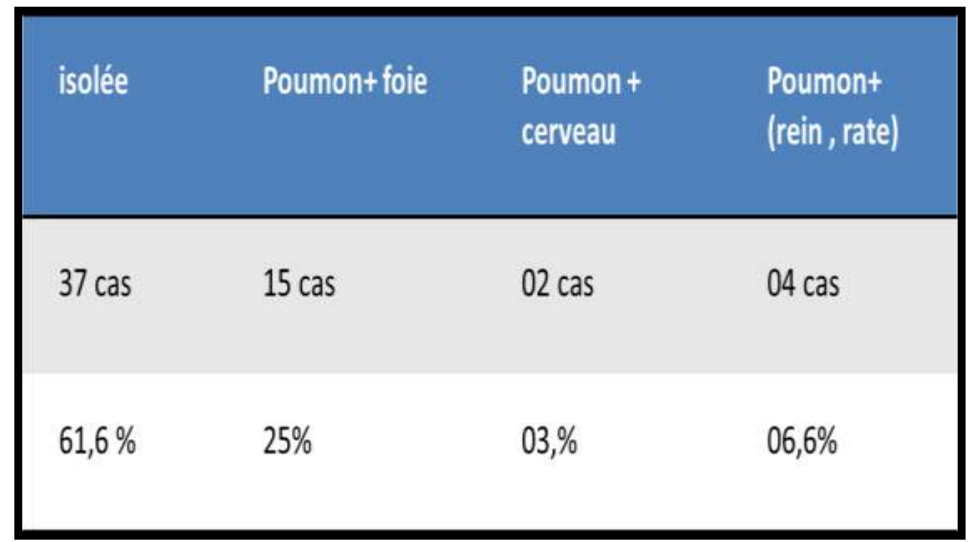

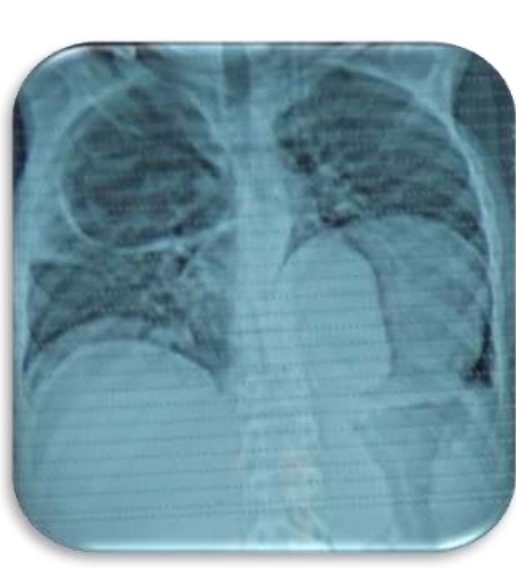
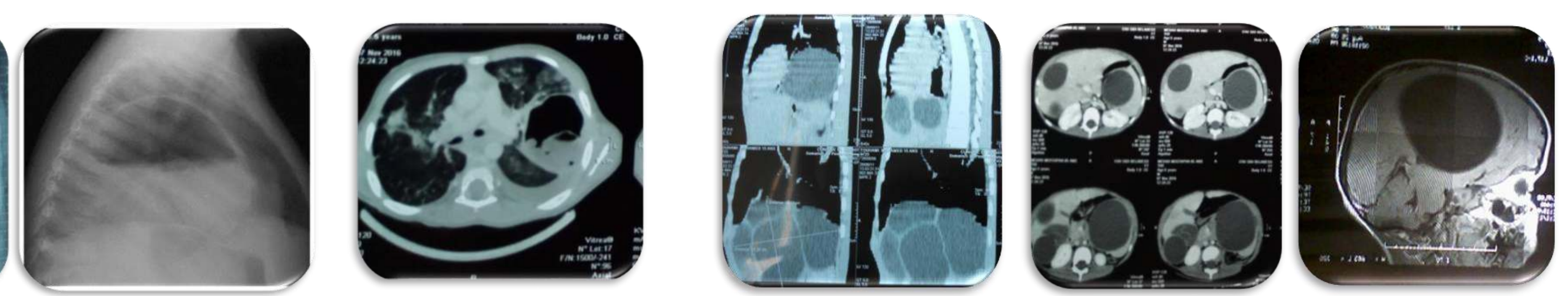

Fig. $(1,2,3,4,5,6):$ Rx and TDM KHP + associated locations
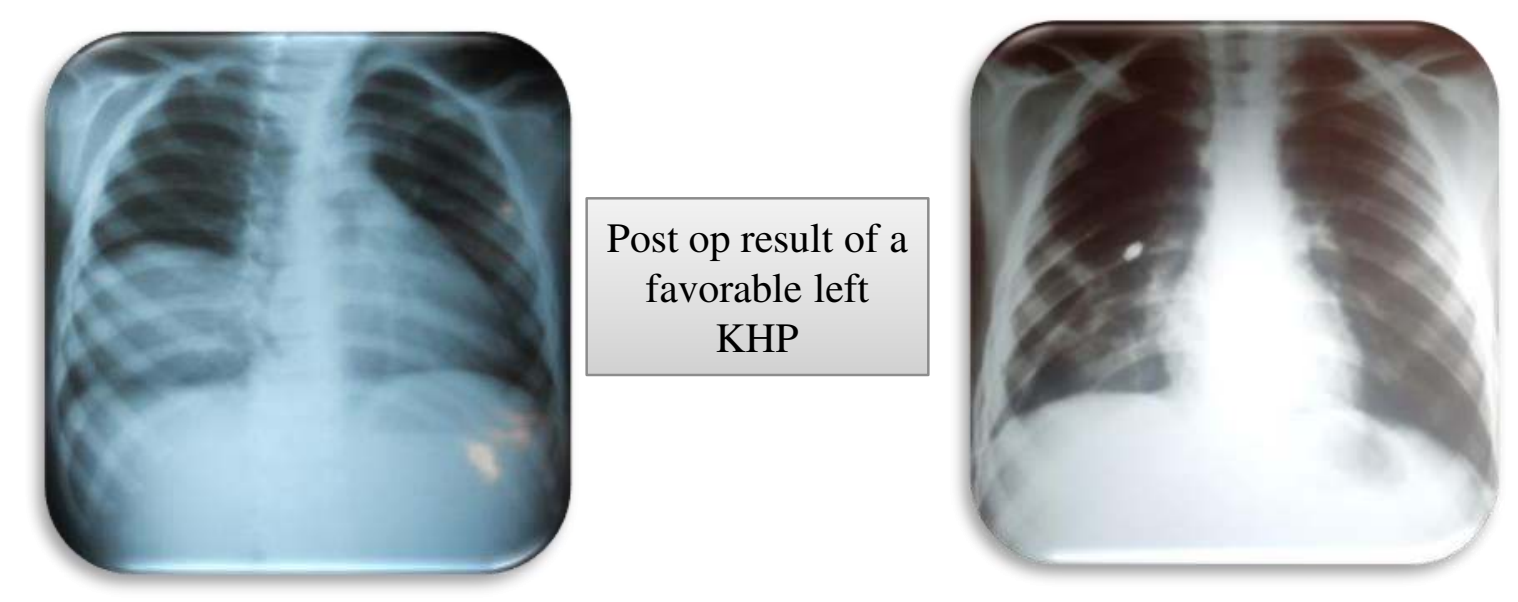

Clinicat case: $(* *)$

Child aged 07 operated for a giant right KHP at Day 15 post op PNO encysted (pachy pleuritic) recurrent Iterative thoracotomy, pleural decortication + fistula closure + double drainage
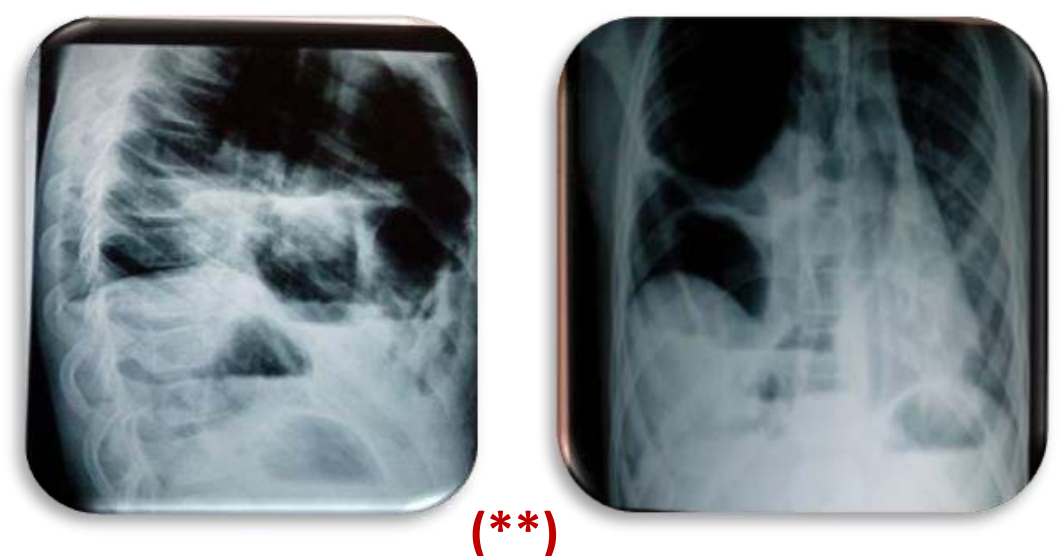

Références 